\title{
3D Skin Texture Analysis: A Neural Network and Photometric Stereo Perspective
}

\author{
Shahzad ANWAR*, Lyndon N. SMITH and Melvyn L. SMITH \\ Machine Vision Laboratory, University of the West of England, Bristol, UK.
}

\begin{abstract}
Cancerous skin lesions often exhibit irregular and non-axisymmetric morphologies and 3D textures, which are difficult to detect using existing techniques. Consequently, this paper describes the employment of a novel technique incorporating Multilayer perceptron (MLP) Neural Network (NN) and photometric stereo (PS) techniques for the analysis of complex lesions. The analyses of surface normal data (tilt and slant angles), for measurement of the degree of 3D skin surface disruption can provide potentially useful indicators for melanoma. Here this was achieved by replacing the axisymmetric 3D hemispherical profile with NN models of irregular lesion morphologies. PS was used to recover surface data and a NN was used to model the underlying forms of complex morphologies and irregular 3D textures. Initially, application of the method to three types of lesion resulted in an average difference (in slant angle) between the NN output and the actual surface normal of: 1.02, 2.11 and 2.86 degrees for axisymmetric, irregular and complex lesions, respectively. The proposed method performance was significantly better when compared to other methods. The experimental study performed shows the effectiveness of the proposed method, with ROC area under the curve of $84 \%$ to $88 \%$.
\end{abstract}

Keywords: 3D clinical imaging, Photometric Stereo, bump map Neural Network.

\section{Introduction}

Melanoma is a complicated form of skin cancer and the increasing rate of occurrence of this disease is a worldwide concern. MM spreads in two stages (i) superficial spreading, where the tumour increases its size within the epidermis (causes in a skin surface disruption) and (ii) perpendicular growth phase, where the tumour begins to develop into the dermis. The growths in these two levels (i.e. dermis and epidermis) are determined by the Clark level [1]. The dermatologist generally incorporates the famous, (A) Asymmetry, (B) Border irregularity, (C) Colour variation and (D) Dimension (ABCD) rule, as an aid in the identification of melanoma [2]. The cardinal characteristic observed in skin lesion that establish to be melanoma, is a change observed over a period of time (often in weeks), and this time scale is significant. For instance, if a lesion rapidly develop that it double its size within about 45-60 days, it is often an inflammatory condition, alternatively, if a lesion grow leisurely that the patient is unsure of any changes the lesion is often benign [3]. The consultant's advice should always be sought, regardless of the lesion's growth rate. Mass screening for this disease have been advocated for an effective treatment [4], however, the golden standard for the treatment of melanoma would still be the biopsy assessment.

It is well known that human skin is covered with a fine texture, which is an important visual indicator in describing and assessing skin surface [5]. Texture depends upon a number of factors such as spatial relation between primitive texture element, scale, and/or orientations [6]. Skin texture (often) gradually changes and becomes apparent once the overt change occurs. One of the major problems dermatologists often face during examinations is distinguishing texture of various skin lesions. Consequently, there have been many attempts to incorporate the role of computer models to accomplish this recognition task [7-9].

Previous research on 2D lesion characteristics [10] has been beneficial for classifying melanoma and benign lesions; however, only limited research has been performed on 3D lesion characteristics, perhaps due to the limited capabilities for acquiring 3D skin textural data. To address this, Ding et.al. [11] proposed a photometric stereo technique to capture lesion surface 3D textures, which are then analysed (in the form of a 'bump map' of surface gradients), for generating indicators of possible MM in human skin. In Ding's method, a 2D isotropic distribution, which is uniformly distributed with respect to the distribution centre of the lesion, was chosen as the function for generating a skin slant/tilt pattern model. A series of simulated 2D Gaussian profiles were employed to model the lesion surface in order to minimise the error between the recovered bump map of the lesion and the synthetic model.

* corresponding author shahzad2.anwar@live.uwe.ac.uk 
However, these multiple surfaces are computationally an expensive solution for analysing the lesion morphology. Another limitation was that the 3D surface generated from a 2D Gaussian was limited to regular (axis-symmetric) shapes; whereas cancerous lesions often exhibit irregular morphologies and 3D textures. Therefore, Ding's model could perhaps have some limitations in the analysis of 3D irregular (complex) lesions.

These limitations are overcome in the present study by making use of the non-prescriptive, (i.e. data-driven rather than assuming any particular function) and non-linear modelling capabilities of neural networks (NN). Therefore the aim of the study is to combine NN and PS to develop a non-invasive machine vision technique for the identification of MM. Receiver operation characteristic $(\mathrm{ROC})$ analysis was performed to evaluate the performance of the proposed method and the results were compared with existing methods. The experimental study performed shows the effectiveness of the proposed method, with overall ROC accuracy of $84 \%$ to $88 \%$.

The remainder of this paper is organised as follows. Section 2 presents the materials and methods employed, including the establishment of the NN design, training and its testing methodologies. Section 3 explains the experimental procedure, whilst results and discussion are presented in section 4. Finally conclusions are drawn in section 5 .

\section{Materials and method}

This section firstly introduces the PS technique, which was employed for image capture and was necessary to record and measure the bump map information. The facility for data acquisition is based on 6-light PS technique. The mathematical foundation of standard PS is introduced at the beginning. The subsequent section presents the network design strategy and its learning and training rules, followed by its testing procedure.

\subsection{The theory of photometric stereo}

The critical influence of light direction on the nature of acquired images has led to the investigation of the PS technique [12]. This was later explored in the generation of 3D data which can subsequently be analysed in much depth [13]. PS presents a great advance to the conventional photographs, which are very susceptible to imaging noise. The theory of PS is to employ a surface reflectance model to recover the surface physical properties (i.e. orientations and reflection). At least three images, each captured from a fixed point under different illumination, are required for dimensional orientation. The direction of the light, which is a single moveable source, is defined by two angles (i.e. slant \& tilt). Slant is the angle between the illumination vector and the $z$-axis and tilt is the angle between $x$-axis and the projection of the illumination vector onto the $x-y$ plane. A standard PS geometry with its slant and tilt angles is shown in Fig. 1.

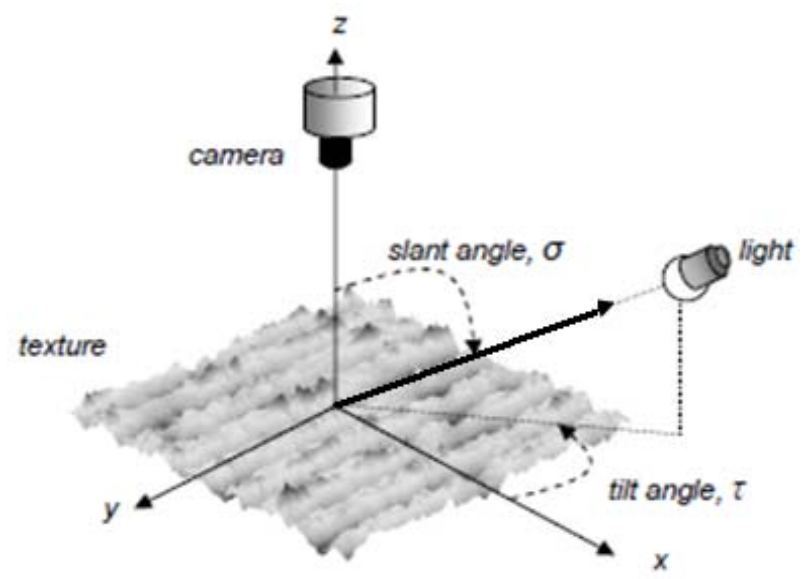

Fig. 1. PS geometry of a surface normal vector with its tilt and slant angles.

In the PS method an assumption is made that the object's surface is Lambertian [14]. Lambertian is a surface with perfectly matte properties, which means that these surfaces reflect light with equal intensity in all directions, and hence appear equally bright from all directions. For a given surface the brightness depends only on the angle $\theta$ between the direction of the light-source $L$ and the surface normal $N$. If an approximately flat texture plane coincides with $x-y$ plane, the surface $S$ can be described as a height function as follows:

$$
z=S(x, y)
$$


The surface gradients in the $\mathrm{x}$ and the $\mathrm{y}$ directions for a facet on such a surface are therefore as $p=\partial_{z} / \partial_{x} q=\partial_{z} / \partial_{y}$ respectively, where $-1<p, q<+1$. Two tangents perpendicular to this facet can

then be written in vector form as $[1,0, p]$ and $[0,1, q]$. The vector normal to the facet, $N$, is found by taking the cross-product of these tangents. $N=[p, q,-1]$. Once normalised this becomes:

$$
N=\frac{1}{\sqrt{p^{2}+q^{2}+1}}[p, q,-1]
$$

If the facet is illuminated by a light-source, the unit illumination vector $L$, which points away from the surface is written:

$$
L=l_{x}, l_{y}, l_{z}
$$

Defined in terms of a polar co-ordinate system this becomes:

$$
L=(\cos \tau \sin \delta, \sin \tau \sin \delta, \cos \delta)
$$

Where is $\tau$ tilt angle and $\delta$ is the slant angle. For an ideal Lambertian surface, the image irradiance equation can be expressed as:

$$
i=\rho . \frac{-p \cos \delta \sin \tau-q \sin \delta \sin \tau+\cos \tau}{\sqrt{p^{2}+q^{2}+1}}
$$

Where ' $\rho$ ' is the surface reflection rate (albedo). The employment of tilt and slant angles inherently gives more physical meaning compared to surface normals. Consequently, a neural network based approach to predict the gradients of the surface normals vector (i.e. tilt angle in $x-y$ axis directions) and slant in z-axis direction has been proposed and demonstrated for skin textural data examination.

\subsection{Skin Analyser for clinical data collection}

The Skin Analyser was commissioned at the Department of Dermatology at Frenchay Hospital NHS Trust Bristol, UK for clinical data collection. The skin lesion data examined in the present study was recorded with the Skin Analyser [16] which is shown in Fig. 2. It is a handheld device composed of an IEEE 1394 digital camera (AVT Marlin, F-046C), high-resolution compact lens (Schneider, 1.4/23 mm + extension tube) and six high power SMD white light LEDs (Nichia, E-NSC455). The image capturing mechanism works with customised software and can reach a resolution of 23 microns. The PS captured images have spatial resolution of 1000 x 1000 pixels.

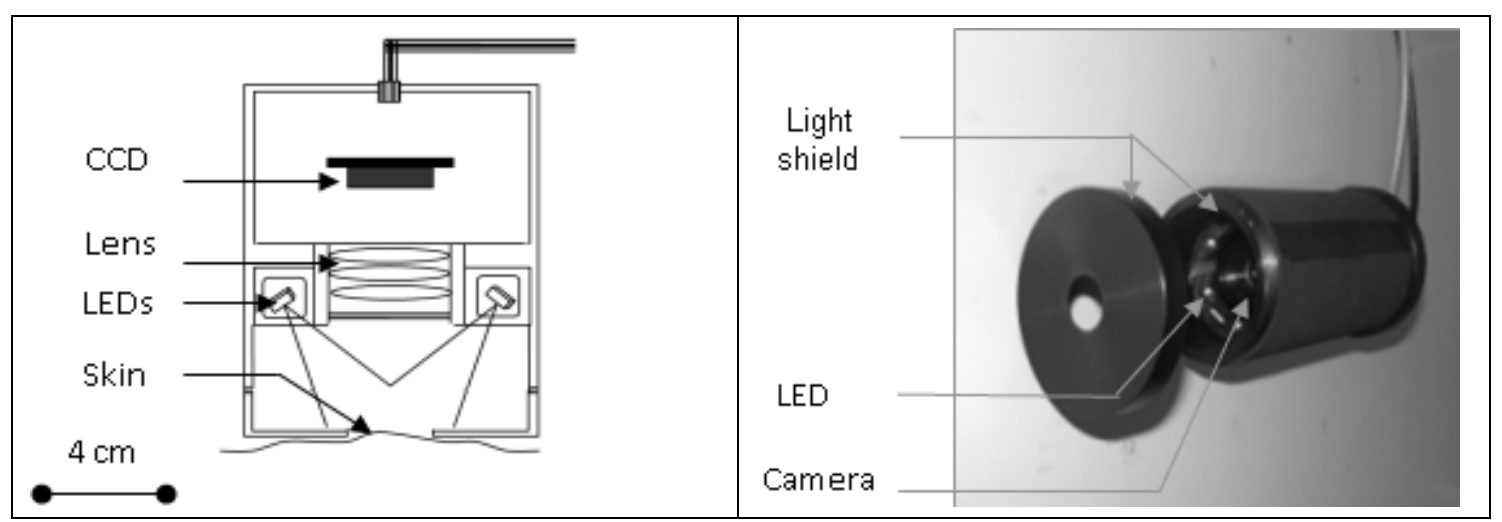

Fig. 2. Left: Schematic to scale; Right: the handheld colour PS device known as the 'Skin Analyser' (Mark I) [15].

The theory of this device is based on six-light PS by capturing six images each under a differently-positioned illuminant. Among the six images, three images are necessary to solve the irradiance equation as required by a standard three-light PS. The three remaining images have redundant information that can be utilised for pixels having specular highlights and shadows. As a result, accurate surface normal data can be obtained even in the presence of strong specularities and shadows. This is in contrast to the conventional photographs which are very susceptible to imaging noise such as specularities and shadows.

\subsection{Multilayer perceptron neural network architecture}

The human brain is highly complex, non-linear, and massively parallel computing system. The brain structure consists of approximately 10 billion basic units called 'the neuron' and trillions of interconnections. Inspired by the human brain, neural networks (NNs) emulate brain's biological network, and their usage have been widely established in many applications [16-19]. NNs have the 
ability to learn from data and subsequently offer the desire output. There are many types of neural network in the literature $[20,21]$. The MLP is a feedforward network, where all the connections are from the input to the output [22]. Transfer function represents the activation, which is applied in the network hidden layer. Hard limit transfer functions consist of step function with an output of either 0 or 1 (i.e. true or false) whilst linear transfer function is where the output is proportional to the input. Another type of transfer function is the sigmoid (s-shape curve) comprising a curve with the output range from -1 to 1 . By employing sufficient number of hidden units (neurons), the network could model any decision boundary with arbitrary accuracy, and this concept has been employed in modelling the bump map of 3D lesions. The architectural design follows three basic steps: (i) creating the network, (ii) learning and training algorithm followed by (iii) testing the network on dataset. The choice of MLP architecture in implementation is described in the following sub section.

\section{MLP NN design}

The Multilayer perceptron (MLP) neural network (NN) design was developed to accomplish the objective of 3D lesion modelling in terms of the gradient of the bump map. The MLP has some important properties which are beneficial in recognising patterns in the presence of noise [23, 24]. Non-linear modelling capability was a factor of particular interest whilst choosing MLP NN architecture for our task. When the training set contains considerable noise or inconsistent samples (surfaces irregularities) during the learning phase, the network extracts the underlying model of the set. By carefully controlling the NN learning parameters, the network can be made to generalise (i.e. to model the underlying pattern rather than the actual noise) and this can be verified by consulting the network with a test dataset (samples that are not included in the training set). This is particularly important in practice, particularly when considerable noise is present in the data. The establishment of network architecture involves choosing the number of hidden layers, and hidden layer neurons. Due to the highly non-linear nature of the surfaces, the neurons have sigmoid transfer functions. The network design was the initial step prior to training and testing for accuracy.

\section{MLP NN training}

Once the network was created, the subsequent step was training it on a dataset. The data was split into two sets i.e. training and test data. The bump map consists of $800 \times 800$ vectors and was divided into regions of $50 \times 50$ vectors giving 256 regions. In both cases i.e. melanoma and benign, $75 \%$ data for training (192 regions) was selected to train the network. The training dataset was randomly chosen from the whole dataset. Various training algorithms are reported in the literature, such as error-back propagation and Levenberg-Marquardt training algorithm (LMA) etc. The learning capability of the LMA is reported to be superior [22] and to have rapid convergence advantages [25]. Therefore, LMA was employed to train the network for 12000 epochs. The stopping principle determines the number of epochs before training of the network is required to be stopped. This usually depends on the sum of the squared errors which are the squared differences between the actual output and the desired output. Training a network involves minimising an error measured across a training dataset which is a function of the weight setting in the MLP. Training is usually carried out until a certain number of epochs (e.g. 12000 epochs) or the errors decrease to a minimum value where the training could be stopped.

\section{MLP NN testing}

After the network had been trained on a training dataset the next step was to test the capability of the network to generate useful outputs. The test data was fed to the network to observe the final output in order to confirm the actual values. This was achieved by employing the total data minus the training set as the data set for training. The test allocation data was $25 \%$ of the whole dataset. The training and testing dataset was randomly chosen from the whole dataset. For the work reported, the designed network deploy 24 neurons (12 neurons in each layer) to model the gradient of the bump map. The proposed model is capable to model the lesion's 3D topography (in terms of gradient of the bump map), thereby enabling quantification of the degree of deviation on surface.

\section{Experimental procedure}

Once the Skin Analyser was employed to record clinical data in hospital, the subsequent step was data acquisition, which was performed at the Machine Vision Laboratory at UWE Bristol. The MLP was implemented in MATLAB ${ }^{\circledR}$ ver. 7.

The clinical data investigated in this project has been acquired in regular clinical trials. Overall, 77 skin lesion categorised into two classes (i.e. benign and melanoma) were incorporated for examination. 
The images were captured during routine clinical examinations, to reflect a prior probability of the routine diagnosis in a specialised dermatology clinic. In the database, 15 lesions are categorised as malignant melanoma, whilst 62 lesions are reported as benign. When conducting clinical trials, few essential preliminary guidelines were followed. These include hair removal in either case (i) from lesion and (ii) from the lesion surrounding. The hair removal step was necessary due to the reason that, hair (on the lesion or on the skin) is outliers to colour and texture. They exhibit dissimilar colour and texture information from the skin background and/or lesion. Lee [26] proposed three step software based solution for hair removal. This is reported as (i) localising hairy regions (ii) replacing the hair pixel with nearby non-hair pixel and (iii) smoothing the remaining noise. Although, the software solution is convenient and effective, however, the quality of a recovered skin image captured with hair still cannot matches with those images, obtained with following the essential hair removal process.

The PS captured images of benign lesion and melanomas as shown in Fig. 3 were having resolution of $1000 \times 1000$ pixels. To avoid extra computation, we cropped the image to $800 \times 800$ pixels, thus result in a lesion with slight surrounding of healthy skin. Once data acquisition has been performed, the next step was to differentiate between lesion and the background (i.e. segmentation). There is a significant difference between the object and the background in terms of the gradients; therefore, the process was relatively simple. A threshold value of $3^{\circ}$ for tilt angle was employed to separate the object from the background.

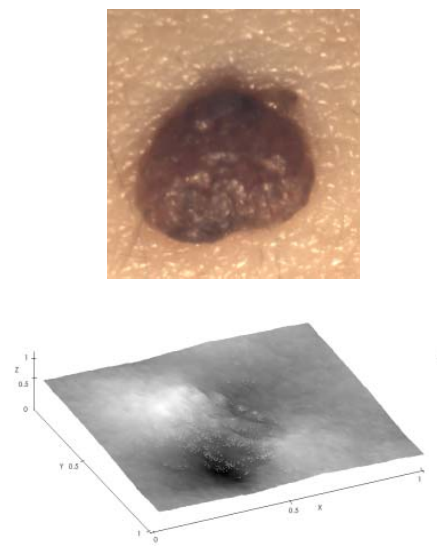

(a)

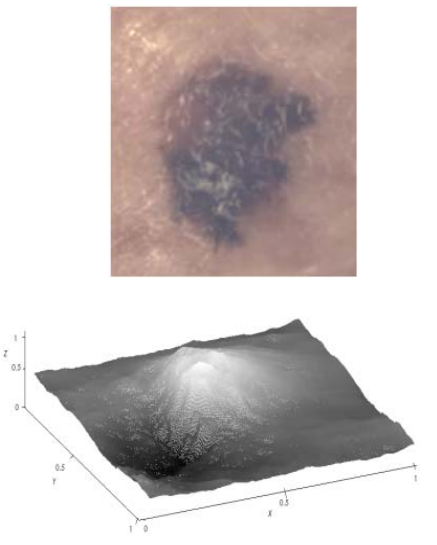

(b)

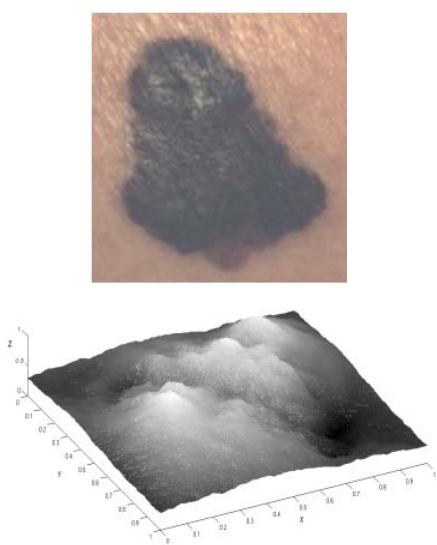

(c)

Fig. 3 (a) Benign lesion (b) melanoma and (c) irregular melanoma. (2D data shown at Top and 3D at bottom).

The analysis of the surface normal data could also be achieved; by independently analysing the gradient of the surface normal (i.e. tilt and slant angle). The advantage of directly analysing the gradients is noticeable. Firstly, recent advances in Markov random field and statistical analysis have demonstrated that the information within a local region for classification, can achieve better results when compared to large and complex spectral filters [27]. Secondly, surface normal could be separated according into two angles and analysed independently at low level, and finally, skin pattern is a form of texture with high local-similarities, this suggest that spatial information are important in describing the skin texture, for this reason, it would allow efficient analysis of the lesion at low level.

The PS acquired images were processed subsequently the MLP NN were commissioned to calculated the gradient of the bump map (i.e. slant and tilt angles) and these data were utilised for training of a network that had $x$ and $y$ coordinates as inputs and slant and tilt angles as outputs. Once trained, the network was consulted to predict slant and tilt for a given $x$ and $y$ position. At each point on the lesion the network was consulted to predict slant and tilt and the actual slant and tilt was subtracted, giving a measure of surface irregularity. The average irregularities were calculated for the entire set of images.

\section{Results and discussion}

The aim was to test the proposed method on benign and melanoma lesions. It is desirable to investigate a relative simple mole; therefore, a benign lesion as shown in Fig. 3, was incorporated for initial examination. Training the network often takes an extensive amount of time, which generally depend upon the selected training algorithm and the computing machine employed, however, once the network has been trained it may be consulted rapidly. Training involves the associated weight of a network being modified iteratively to reduce the error in between the actual gradient of the bump map and the predicted output. A negative slop in the curve as illustrated Fig. 4 indicates that the network is training on a data and is well progressing 


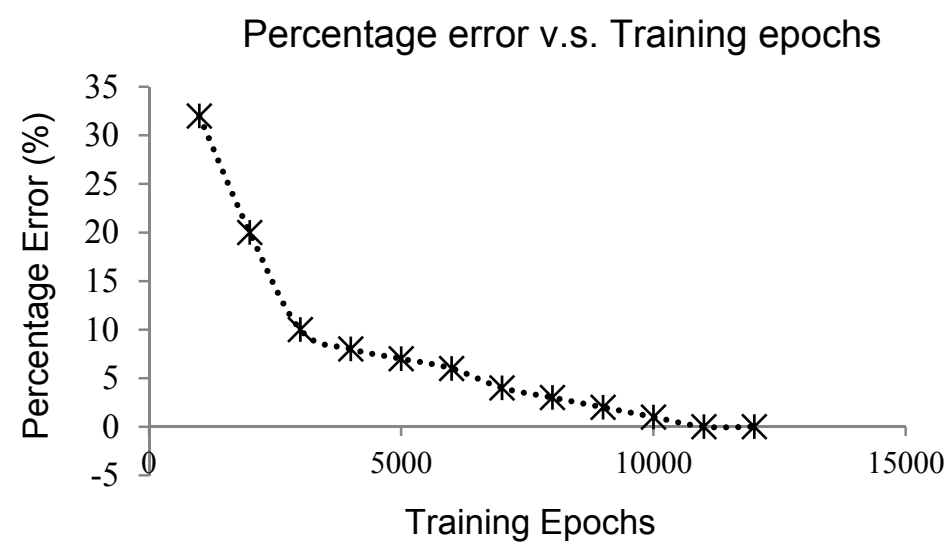

Fig. 4. NN response: The negative slope indicates that with the increase of epochs the error decreases.

In the experimentation, a range of neurons (number varies in between 10 to 28) for a dedicated number of epochs, were deployed to accomplish the objective. For the reported work, the best overall accuracy was achieved via commissioning 24 neurons, which is evident in Fig. 5.

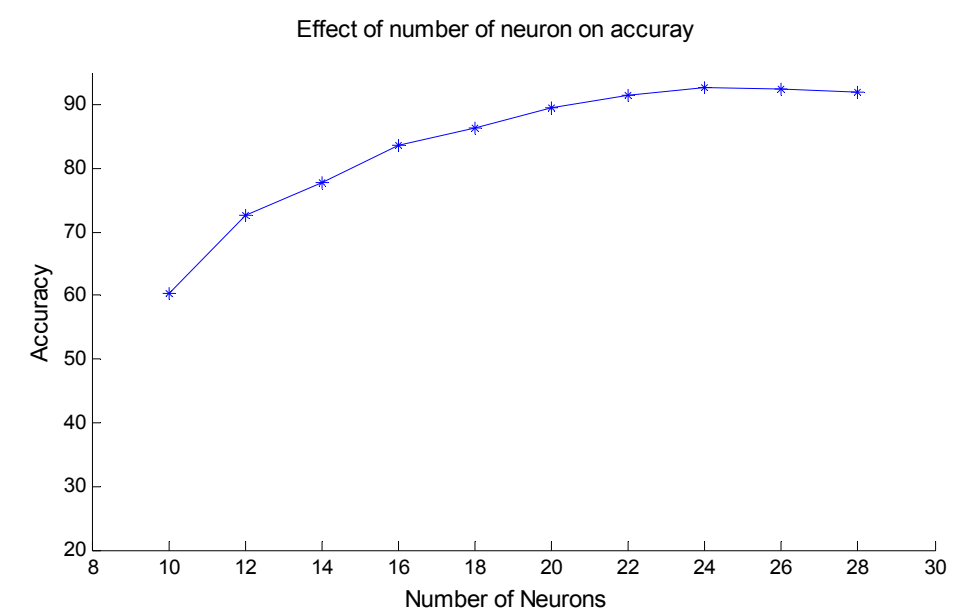

Fig. 5. The effect of accuracy is dependent upon the number of neurons.

Once the network has been trained, the subsequent step was to verify that it generalises well. This was achieved by feeding the test data into the input layers, which was hidden from network during the training process. In the case of benign lesion, the network has promisingly predicted the gradient of the bump map. The regression plot was calculated in MATLAB by employing POSTREG function. The regression value $R=0.94$ for the slant angle is shown in Fig. 6 (a), and confirms its validation. The next step was to incorporate melanoma lesion. The test data for melanoma lesion was fed to the network, and network was able to offer the desired output via predicting the gradients. The network has successfully predicted the desired gradient of the bump map with a regression value of $R=0.92$ which is shown in Fig. 6 (b). The drop in the regression value (as compared to benign lesion) is due to the surface complexity and irregularities in the case of melanoma.

Once the proposed method has demonstrated its performance by modelling various complex lesions, it was necessary to test the capability of the proposed method with other methods for comparison. Therefore, the next section is devoted to performance evaluation. 


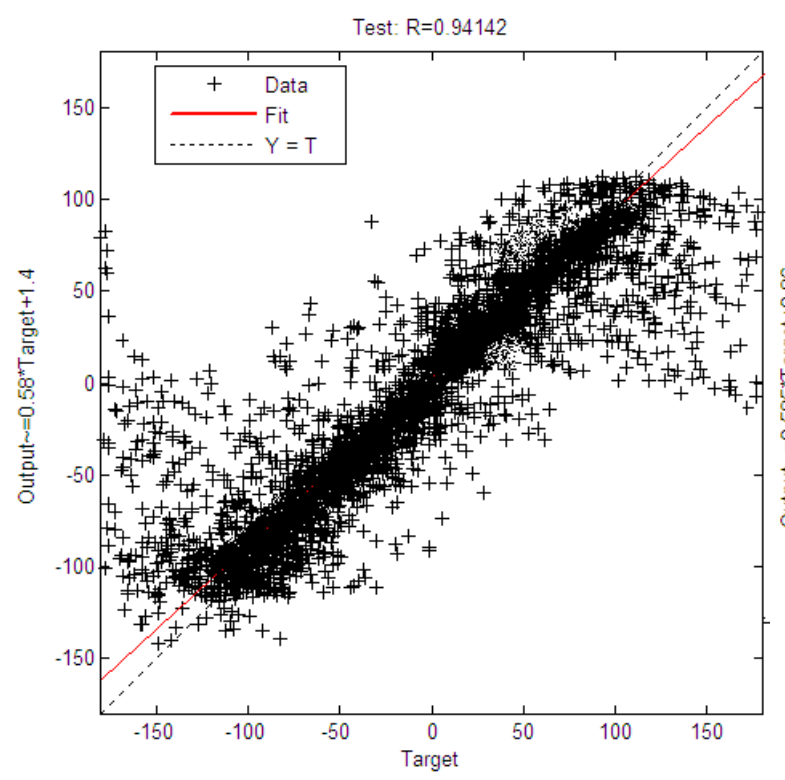

(a)

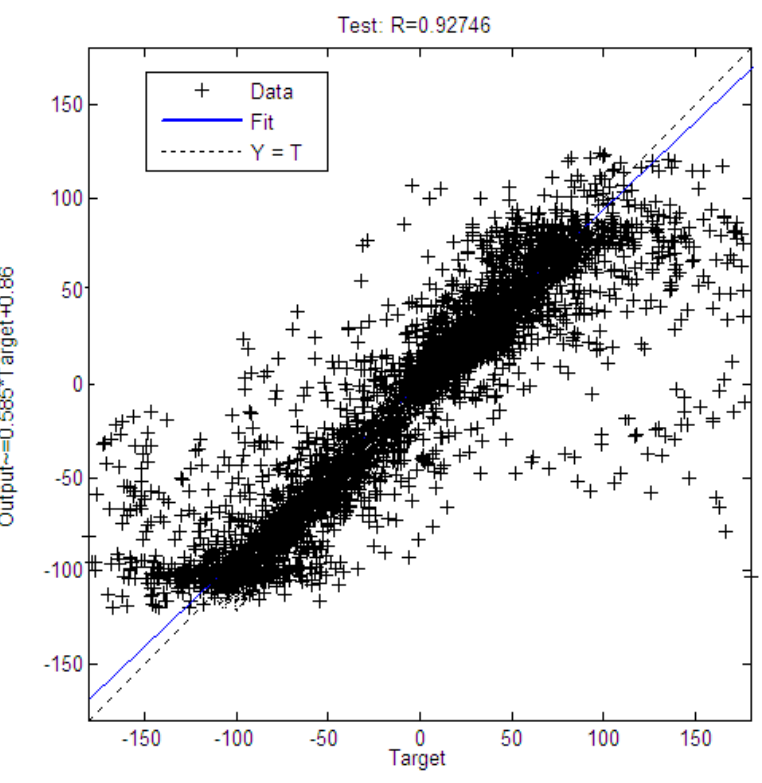

(b)

Fig. 6. Slant angle linear regression for NN modelled of lesion profile (a) $R$-value of 0.94142 for benign lesion and, (b) R-value of 0.92746 for irregular melanoma.

\subsection{Performance evaluation: A comparison}

A comparison with Ding's method [11] for three different lesions (a) regular and symmetrical benign lesion, (b) less regular and symmetrical melanoma lesion and (c) irregular and non-axisymmetric (complex) melanoma lesion was undertaken. For each of the three lesions, Ding chose a 2D isotropic distribution which is uniformly distributed with respect to the distribution centre of the lesion, as the function for generating a skin tilt pattern model. As mentioned previously, a series of simulated 2D Gaussian profiles were generated to model the lesion surface in order to minimise the error between the bump map of the wound and the synthetic model. Skin slant and skin tilt angles were obtained from Ding's method and the results were compared with NN predicted slant and tilt angles. Fig. 7 (a,b\&c) show results for benign, less irregular melanoma and irregular melanoma, respectively. The lesion's slant angle is represented with a solid (blue curve), while skin slant angle calculated via Ding's method is represented with red curve. The NN slant angle distribution is represented with dotted (gray) line in Fig. $7 a$.

For the benign lesion as shown in Fig. 7 (a), both methods were able to model the gradients; however, the proposed method showed an improvement over Ding's method where there was relatively more complexity for instance, at pixel positions 100, 200 and 300, respectively. Ding's method was lagging with a mean error value of $2.74^{\circ}$ to $1.02^{\circ}$. Similarly for an irregular and less symmetrical melanoma lesion the performance of the proposed method was considerably better with mean values of $5.17^{\circ}$ to 2.11 ${ }^{\circ}$, as shown in Tab. 1.

Tab. 1. A quantitative assessment with Ding's method.

\begin{tabular}{|l|l|l|l|}
\hline \multirow{2}{*}{} & \multicolumn{3}{|l|}{ Mean value error (in slant angle) } \\
\cline { 2 - 4 } & $\begin{array}{l}\text { Symmetrical and } \\
\text { regular benign } \\
\text { lesion }\end{array}$ & $\begin{array}{l}\text { Non-symmetrical and } \\
\text { less regular melanoma } \\
\text { lesion }\end{array}$ & $\begin{array}{l}\text { Non- symmetrical and } \\
\text { irregular melanoma lesion }\end{array}$ \\
\hline Ding's method & $2.74^{\circ}$ & $5.17^{\circ}$ & $12.63^{\circ}$ \\
\hline Proposed method & $1.02^{\circ}$ & $2.11^{\circ}$ & $2.86^{\circ}$ \\
\hline
\end{tabular}



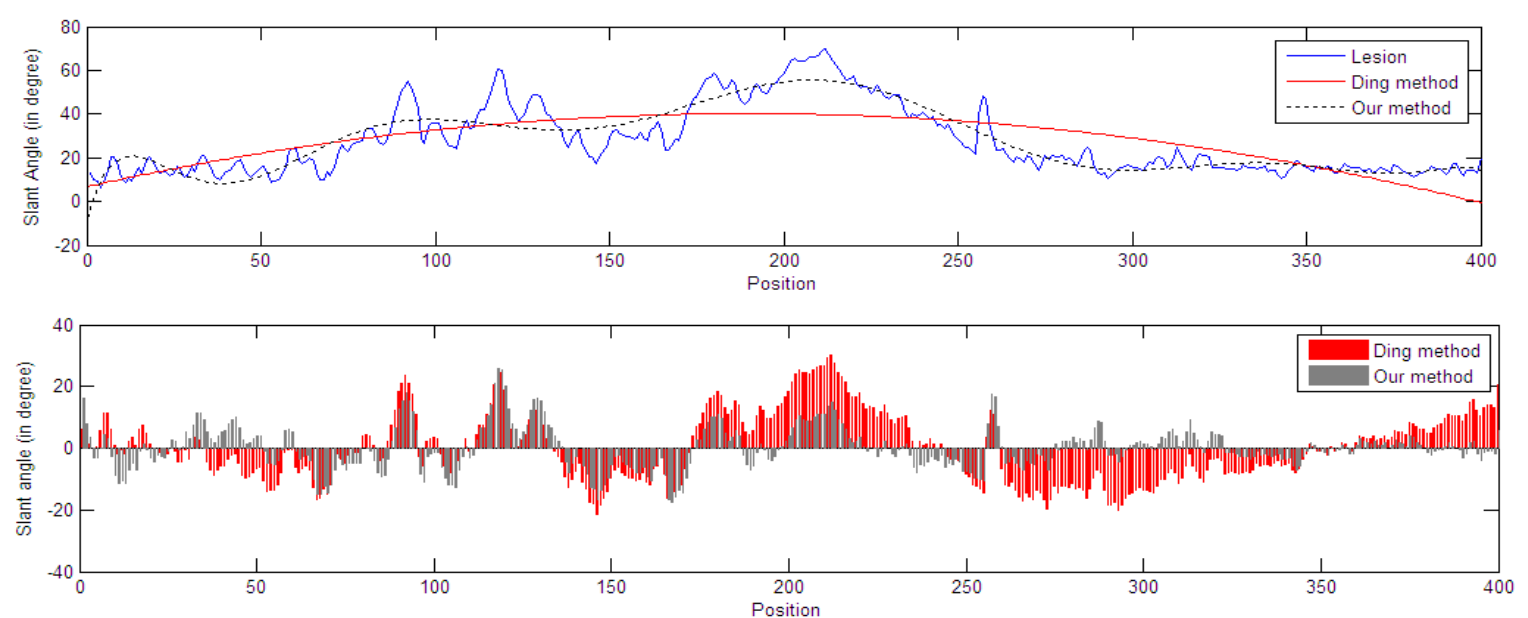

(a)
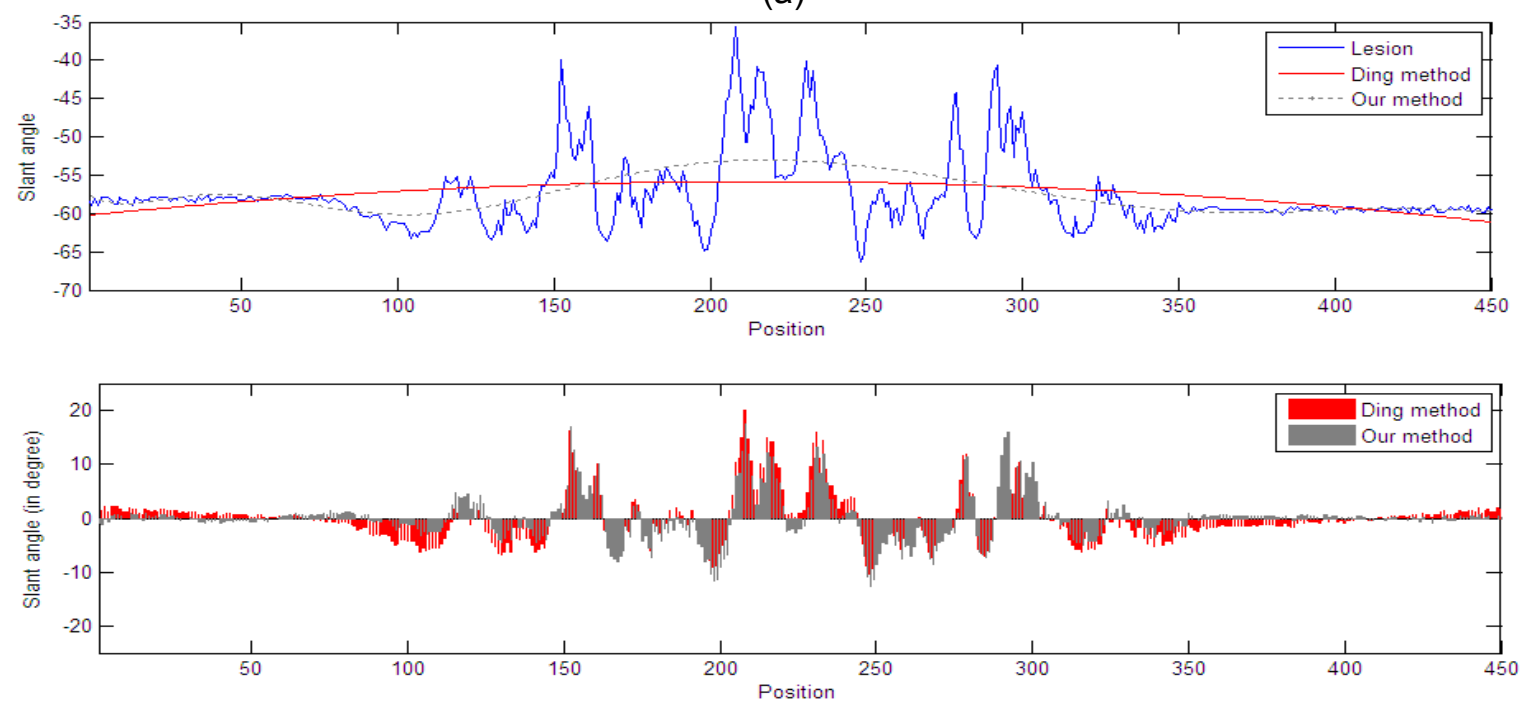

(b)
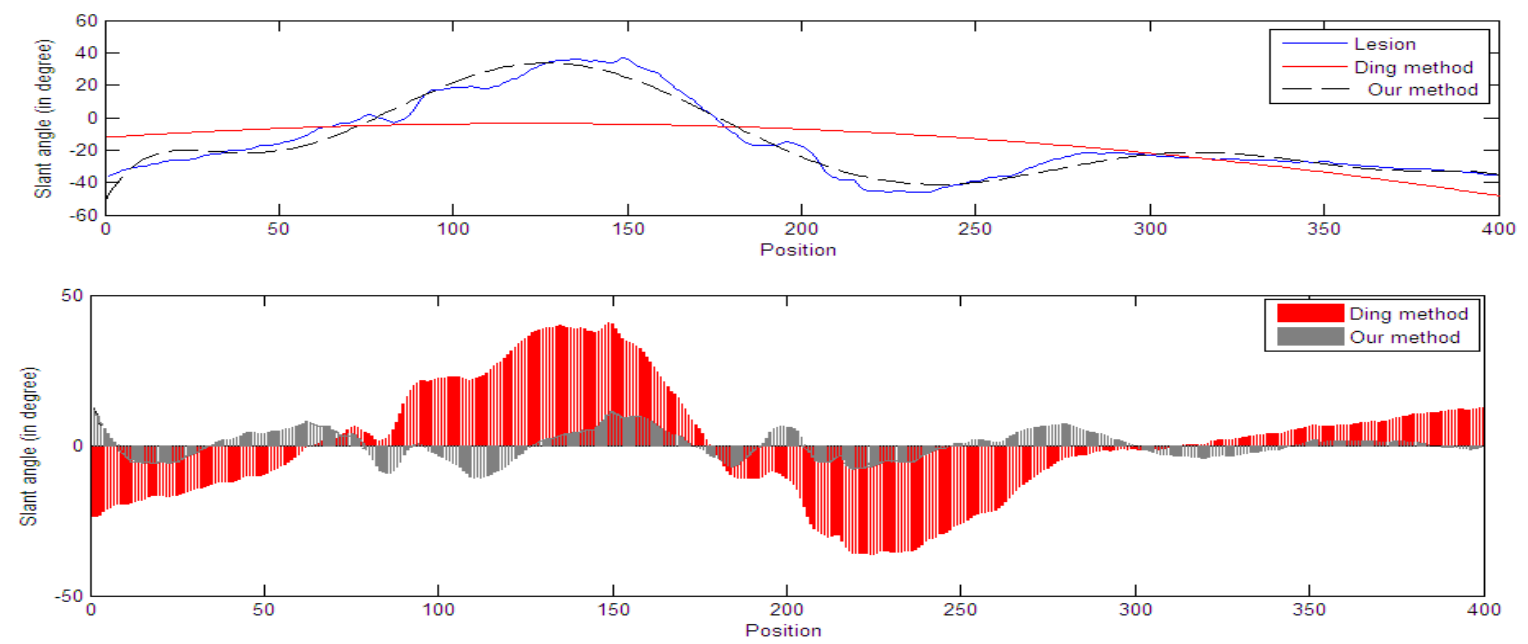

(c)

Fig. 7 (a) Top - Slant angle distribution for regular and symmetrical benign lesion, Bottom - a comparison (error in slant angle) between Ding's method and the proposed method. (b) Top - Slant angle distribution for less regular and symmetrical melanoma lesion, Bottom - a comparison (error in slant angle) between Ding's method and the proposed method. (c) Top - Slant angle distribution for irregular and non-symmetrical melanoma lesion, Bottom - a comparison (error in slant angle) between Ding's method and the proposed method. 
Finally, for an irregular and non-axisymmetric (complex) melanoma lesion, Ding's 2D Gaussian approach was unable to model the lesion profile; however, the proposed method successfully modelled the gradients of the bump map with mean errors of $12.63^{\circ}$ (in Ding's method) to $2.86^{\circ}$ (in the proposed method). This has clearly demonstrated that the proposed method offers much potential in modelling complex and irregular lesions, where previous approaches presented limitations.

A receiver operation characteristic $(\mathrm{ROC})$ test was conducted to evaluate the performance of the proposed method compared to the other methods (i) [10], (ii) [11]. The discriminating power for the proposed method for six skin surface texture features: (i) network predicted skin tilt angle (NPT), (ii) network predicted skin slant angle (NPS), (iii) Dings method, overall skin tilt pattern disruptions or OT, (iv) overall skin slant pattern disruptions or OS, (v) She's method, skin line direction (SLD), and (vi) Skin line variation (SLV) was undertaken. The ROC analysis shown in Fig. 8, was conducted for performance evaluation. The proposed method has significantly improved the ROC curve value as shown in Tab. 2 (SLD and SLV were employed by She, with OT and OS by Ding).

Tab. 2. ROC Analysis (\% age accuracy)

\begin{tabular}{|lc|l|}
\hline Skin Line Direction (SLD) & {$[10]$} & $59 \%$ \\
\hline Skin Line Variation (SLV) & {$[10]$} & $64 \%$ \\
\hline Overall Tilt Angle (OT) & {$[11]$} & $71 \%$ \\
\hline Overall Slant Angle (OS) & {$[11]$} & $75 \%$ \\
\hline Network Predicted Tilt (NPT) & [proposed method] & $\mathbf{8 4 \%}$ \\
\hline Network Predicted Slant (NPS) & [proposed method] & $\mathbf{8 8 \%}$ \\
\hline
\end{tabular}

The proposed non-invasive scheme based on neural network and machine vision techniques explores the analysis of surface normal data (tilt and slant angle), which offers valuable and potentially complementary 3D indicators, in the form of the degree of the 3D skin surface disruption, for the diagnosis of melanoma. There have been increasing demands for a non-invasive computer vision system which offer benefits such as, detailed descriptions of skin. Such a system is a combination of multi-disciplinary engineering, for instance, computer vision, machine learning, neural networks and texture (skin texture) biomedical engineering. These systems are capable of converting qualitative interpretations of the physical and textural characteristics into quantitative results. Subsequently the results could either be translated into a specific suggestion for diagnostic procedures, or to make a judgment over the malignancy of a particular lesion. The diagnoses are often heavily dependent on the accuracy and the accuracy varies according to the clinician experience. Whilst diagnosing the melanoma at an early stage, dermatologists generally incorporate features such as the ABCD rules for visual examination; these are subjective and might lead to false diagnosis. Due to the wide range of the diseases, a clear distinction between a melanoma and a benign lesion is very challenging [28]. An alternative solution is that lesion's images may be collected (in clinics) at regular intervals and a non-invasive neural network based machine vision technique could be employed to assist with the early identification of this deadly disease. Therefore, employing a machine vision detection method mainly at primary health care will not only able to reduce the number of expensive unnecessary referrals (currently occurring in the NHS), but will also assist with the identification of melanoma.

\section{Conclusions}

This article has proposed and demonstrated a novel photometric stereo and neural network based approach for modelling irregular and non-axisymmetric surfaces (in term of the gradient of the bump map). This research investigates the analysis of surface normal data, which offers valuable and potentially complementary 3D indicators, in the form of degree of 3D skin surface disruptions, for the identification of melanoma. The PS technique was employed to record 2D/3D data, and the neural network to predict the gradients of the surface normal vectors. The network has initially predicted the gradient for multiple hemispheres, subsequently to test the performance, the network was tested to predict the gradients for real clinical images i.e. a benign and two melanoma lesions, and the performance of the proposed method was compared with an establish method. It is to be noted that due to the wide range of dermatological diseases, a clear distinction between melanoma and benign is very demanding. Consequently, employing a single technique to accomplish a fully accurate diagnosis system is very challenging. Hence, further improvement is always being sought in the form of additional dimensional information or feature descriptors. 


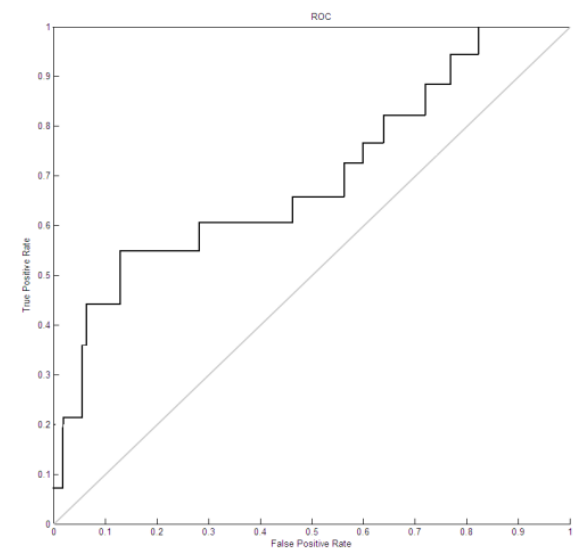

(a)

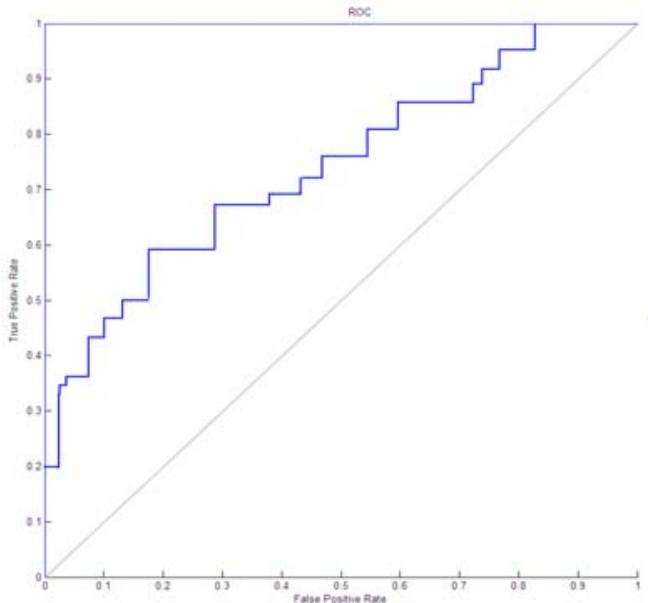

(c)

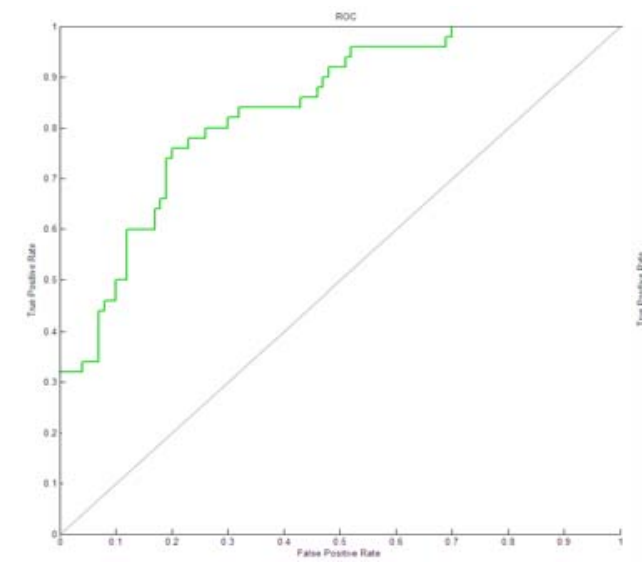

(e)

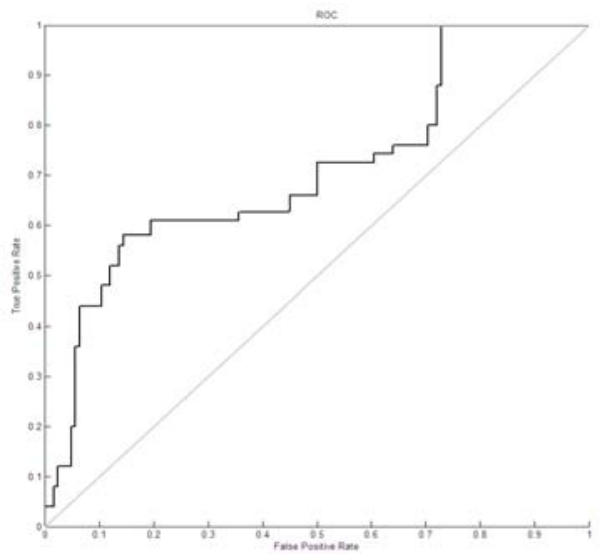

(b)

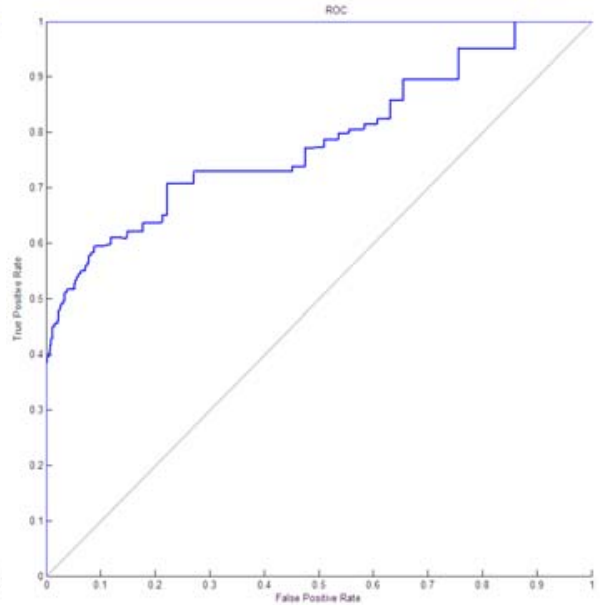

(d)

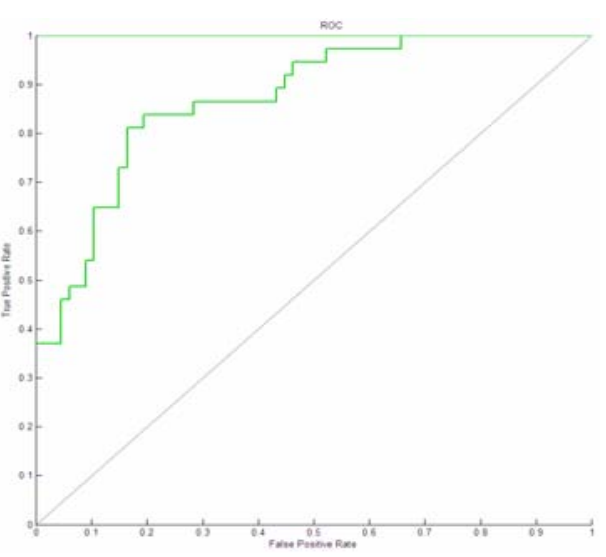

(f)

Fig. 8. ROC curve for (a) SLD, (b) SLV, (c) OT, (d) OS, (e) NPT, and (f) NPS.

\section{References}

1. J. Clark, L. From, E. Bernardino, and M. Mihm, (1969): "The histogenesis and biologic behaviour of primary human malignant melanomas of the skin," Cancer Research, vol. 29, pp. 705-726.

2. Franz N. et al., (1994): "The ABCD rule of dermatoscopy: High prospective value in the diagnosis of doubtful melanocytic skin lesions," Journal of the American Academy of Dermatology, vol. 30, no. 4 , pp. 551-559.

3. A. Balsari, G. Tona, M. P. Colombo, G. Fossaati, and G. Parmiani, (2006): "Control of human melanoma growth in nude mice by autologous allo-activated peripheral blood lymphocytes," International Journal of Cancer, vol. 38, no. 6, pp. 923-927.

4. R. Hamidi, D. Peng, and M. Cockburn, (2010): "Efficacy of skin self-examination for the early detection of melanoma," International Journal of Dermatology, vol. 49, no. 2, pp. 126-134. 
5. L. Smith, M. Smith, A. Farooq, J. Sun, Y. Ding, and R. Warr, (2011): "Machine vision 3D skin texture analysis for detection of melanoma," Sensor Review, vol. 31, no. 2, pp. 111-119.

6. J. Zhang, M. Marszalek, S. Lazebnik, and C. Schmid, (2006,): "Local features and kernels for classification of Texture and object categories: A comprehensive study," in Computer Vision and Pattern Recognition Workshop, pp. 13-20.

7. B. Ginneken, B. Romeny, and M. Viergever, (2001): "Computer-aided diagnosis in chest radiography: a survey," IEEE Transactions on Medical Imaging, vol. 20, no. 12, pp. 1228-1241.

8. K. Nakamura et al., (2000): "Computerized analysis of the likelihood of malignancy in solitary pulmonary nodules with use of artificial neural networks," Radiology, vol. 214, no. 3, pp. 823-830.

9. L. Wei, Y. Yang, R. Nishikawa, and Y. Jiang, (2005): "A study on several machine-learning methods for classification of malignant and benign clustered microcalcifications," IEEE Transactions on Medical Imaging, vol. 24, no. 3, pp. 371- 380.

10. Z. She, Y. Liu, and A. Damatoa, (2007): "Combination of features from skin pattern and ABCD analysis for lesion classification," Skin Research and Technology, vol. 13, no. 1, pp. 25-33.

11. Y. Ding, L. Smith, M. Smith, J. Sun, and R. Warr, (2009): "Obtaining malignant melanoma indicators through statistical analysis of 3D skin surface disruptions," Skin Research and Technology, vol. 15, no. 3, pp. 262-70.

12. R. Woodham, (1980): "Photometric method for determining surface orientation from multiple images," Optical Engineering, vol. 19, no. 1, pp. 139-144.

13. V. Argyriou and M. Petrou, (2010): "Recursive photometric stereo when multiple shadows and highlights are present," in IEEE Conference on Computer Vision and Pattern Recognition, no. 1, pp. 1-6.

14. J. Lambert, (1760): Photometria sive de mensure de gratibus luminis, colorum umbrae. Eberhard Klett,

15. J. Sun, M. Smith, L. Smith, R. Dabis, C. Harland, and J. Bamber, (2007): "Reflectance of human skin using colour photometric stereo - with particular application to pigmented lesion analysis," Skin Research and Technology, vol. 14, no. 2, pp. 173-179.

16. S. Anwar, L. Smith, and M. Smith, (2011): "3D Texture Analysis using Co-occurrence Matrix Feature for Classification," in 4th York Doctoral Conference on Computer Science (YDS11), York, UK, pp. 05-14.

17. B. Daya, S. Khawandi, and M. Akoum, (2010): "Applying neural network architecture for inverse kinematics problem in robotics," Journal of Software Engineering and Applications, vol. 3, no. 3, pp. 230-239.

18. H. Cheng, X. Shi, R. Min, L. Hu, X. Cai, and H. Du, (2006): "Approaches for automated detection and classification of masses in mammograms," Pattern Recognition, vol. 39, no. 4, pp. 646-668.

19. O. Singh, N. Singla, and M. Sharma, (2011): "Optimization of Feedforward neural network for audio classification systems," International Journal of Advanced Engineering Sciences, vol. 7, no. 1, pp. 98-102.

20. G. Corsini, M. Diani, and R. Grasso, (2003): "Radial Basis Function and Multilayer Perceptron neural networks for sea water optically active parameter estimation in case II waters: a comparison," International Journal of Remote Sensing, vol. 24, no. 20, pp. 3917-3932.

21. J. Hopfield, (1982): "Neural networks and physical systems with emergent collective computational abilities," in National academy of sciences, vol. 79, no. 8, p. 2554.

22. I. Aizenberg and C. Moraga, (2007): "Multilayer feedforward neural network based on multi-valued neurons (MLMVN) and a backpropagation learning algorithm," Soft Computing-A Fusion of Foundations, Methodologies and Applications, vol. 11, no. 2, pp. 169-183.

23. C. Jung, S. Ban, and S. Jeong, (2010): "Input and output mapping sensitive auto-associative multilayer perceptron for computer interface system based on image processing of laser pointer spot," Lecture Notes in Computer Science, vol. 6444, pp. 185-192.

24. C. Lee and T. Hsiung, (2009): "Sensitivity analysis on a multilayer perceptron model for recognizing liquefaction cases," Computers and Geotechnics, vol. 36, no. 7, pp. 1157-1163.

25. [26] M. Lourakis and A. Argyros, (2005): "Is Levenberg-Marquardt the most efficient optimization algorithm for implementing bundle adjustment?," in 10th IEEE International Conference on Computer Vision, vol. 2, pp. 1526-1531.

26. T. Lee, V. Ng, R. Gallagher, A. Coldman, and D. McLean, (1997): "A software approach to hair removal from images," Computers in Biology and Medicine, vol. 27, pp. 533-543.

27. M. Varma and A. Zisserman, (2003): "Texture classification: Are filter banks necessary?," in IEEE Computer Society Conference on Computer Vision and Pattern Recognition, pp. 691-698.

28. C. Morton and R. Mackie, (1998): "Clinical accuracy of the diagnosis of cutaneous malignant melanoma," British Journal of Dermatology, vol. 138, no. 2, pp. 374-378. 